\title{
Focused Ion Beam tomography of WC-Co cemented carbides
}

E. Jiménez-Piqué ${ }^{1,2}$, M. Turon-Vinas ${ }^{1,2}$, H. Chen ${ }^{1}$, T. Trifonov ${ }^{2}$, J. Fair ${ }^{3}$, E. Tarrés $^{3}$ and L. Llanes ${ }^{1,2}$

1 Material Science and Metallurgical Department (CIEFMA). Barcelona School of Engineering (EEBE), Universitat Politècnica de Catalunya. Barcelona. SPAIN

2 Center for Research in Nanoengineering (CRnE). Campus DiagonalBesós. Universitat Politècnica de Catalunya. Barcelona. SPAIN

3 Sandvik Hyperion,Coventry CV40XG,UnitedKingdom

\begin{abstract}
The microstructure of three different grades of WC-Co cemented carbides has been reconstructed in three dimensions after sequential images obtained by focused ion beam. The three-dimensional microstructual parameters are compared against the well-know two-dimensional parameters of grain size, phase percentages and mean free path. Results show good agreement with the exception of individual grain recognition, which could not be univocally segmented. In the case of mean free path, the three-dimensional image depicts a more realistic image of the metal interconnections in the composite.

For the grade with coarser grain size, the obtained tomography is translated in a finite element modelling mesh, and elastic residual stresses are estimated from sintering to room temperature. Calculated thermal stresses agree with experimental results and show significant local variations in their value due to the complex microstructure of cemented carbides.
\end{abstract}




\section{Introduction}

WC-Co cemented carbides (hardmetals) are one of the most successful examples of effective implementation of multiphase composites for structural applications [1-3]. Due to the quite different properties of their two constituents: hard ceramic particles embedded in a metallic binder, hardmetals exhibit an excellent combination of hardness, strength and toughness together with unique wear and abrasive resistance. As a consequence, they are frontrunner candidates for tools used in the manufacturing industry as well as for wear-resistant and structural components in a variety of other industrial sectors.

Through the years, continuous improvement on the performance of cemented carbides has been developed on deeper knowledge of deformation, fracture and wear mechanisms operative under service conditions, and their corresponding link to microstructure variables (e.g. Refs. [4-13]). Accordingly, a reliable and precise quantitative metallographic description of hardmetals has then become a mandatory requirement. Within this context, analysis of microstructure-property relationships for these materials has been approached invoking either composite nature, i.e. content and physical dimensions of each phase, or composite assemblage, i.e. phase distribution and interpenetrating degree between both phases. The relevance of the latter for hardmetals is well-established by the satisfactory description of their mechanical and tribological behaviour on the basis of two-phase microstructural parameters: carbide contiguity $\left(C_{W C}\right)$ - first introduced by Gurland in the late 50's [14] - and binder mean free path thickness $\left(\lambda_{C o}\right)$, as they permit an effective rationalization of the combined influence of independently varied single-phase parameters, i.e. carbide grain size $\left(d_{W C}\right)$ and cobalt content $\left(\%_{w t} \mathrm{Co}\right)$. 
Within the framework of quantitative metallography, the direct or indirect experimental measurement of all the above microstuctural parameters for cemented carbides has been usually done in two dimensions (2D), by means of optical, scanning and/or transmission electron microscopy. Although information gathered following this standard methodology has proven to be quite valuable [15-26], emphasis must be placed on the fact that real microstructure of cemented carbides is three dimensional (3D), with grains of WC being polyhedral. Hence, a 2D projection will only give partial information on the two 3D interpenetrating-phase networks characteristic for these materials. In addition, possible defects in the material, either intrinsic to processing or induced under service (e.g. stable grown cracks $[27,28]$ or corrosion pits $[29,30]$ ) will also be 3D in nature and will then interact with the microstructure in a non-planar way too.

There are several advanced techniques to obtain a 3D microstructural characterization for a given material: X-ray tomography, transmission electron tomography, atom probe tomography or focused ion beam (FIB) tomography, among others. Considering the length scale of microstructural features of hardmetals, FIB tomography emerges as a quite interesting technique as it provides reconstructions in the order a few microns in volume and with resolutions in the order of some nanometers, i.e. very suitable for characterization of phases [31], damage [32] or microcracks [33].

There have been some studies utilizing FIB tomography coupled with EBSD to characterize grains of WC-Co materials, as the work of Borgh et al. [34], where the authors characterized the size of grains and checked orientations and equivalent grain size. In this work we expand the study to three different WC-Co grades with varying grain size and cobalt content, with the aim to correlate the obtained results in $3 \mathrm{D}$ with the microstructural measurements in 2D, namely relative volume of ceramic and metallic phases, grain size of WC, and binder mean free path as well as carbide contiguity. In 
addition, the obtained 3D microstructure of the material with coarser grains will be used to model the residual stresses due to the large difference between the thermal expansion coefficients of metallic binder and carbide, aiming to gain understanding of the local stress states of both phases.

The objective of this research is to investigate the relationship between $2 \mathrm{D}$ and $3 \mathrm{D}$ measurements and the possible differences in microstructural parameters, indicating that 3D measurements can yields richer info in some aspects, such as providing a real microstructure mesh to be implemented for more reliable numerical simulation.

\section{Experimental}

Three different grades of WC-Co where chosen for this study with different grain sizes. All materials were made by Sandvik Hyperion, using conventional laboratory based powder metallurgy methods. Powders were initially mixed in $100 \mathrm{~g}$ batches in $0.25 \mathrm{l}$ carbide lined mills using WC and Co metal powders in the appropriate ratio. They were ball milled during 8 hours, and then pressed into compacts of dimensions $25 \mathrm{~mm}$ x $5 \mathrm{~mm}$ x 5mm approximately. Finally, they were sintered in a vacuum furnace at $1410{ }^{\circ} \mathrm{C}$. The samples were labelled as "Medium" (medium carbide size, high cobalt content), "Ultrafine" (submicrometric carbide size, medium cobalt content) and "Nanometric" (grain size lower than $200 \mathrm{~nm}$, low cobalt content). The main characteristics of all three materials are summarized in table 1.

All materials were polished with a decreasing sequence of diamond slurries down to a $3 \mu \mathrm{m}$ size before FIB milling.

The images for the tomography were acquired with a Zeiss Neon 40 Focused Ion Beam (FIB) coupled with a field-emission scanning electron microscope (FE-SEM) column. 
First, the area of interest was coated with a thin platinum layer by a gas injection system to flatten the surface to avoid "curtain effects" and alignment marks were milled at the surface to help with the reconstruction process. Then, a cube of a few microns was milled with a current of $5 \mathrm{nA}$ at $30 \mathrm{kV}$. Images were then taken by sequential polishing with a current of 200pA at $30 \mathrm{kV}$ and acquiring the images between each step. Table 2 summarizes the scale of the volumes reconstructed for each material.

Once the images were taken, the tomographic reconstruction was done with VSG Avizo software. Images were aligned and noise reduction median filters and edge preserving smoothing Gaussian filters were applied to reduce noise and curtain effect and to homogenize the grey levels of each phase. The two different phases (Co and WC) were segmented by histogram-based binarization. Figure 1 summarizes the experimental procedure followed to obtain the tomographic reconstruction.

The Medium grade was also reconstructed by Synopsys Simpleware and latter meshed for finite element simulation with 3DS Abaqus with tetrahedral elements. Simulation of residual stresses was done assuming constant thermal expansion coefficients of $\alpha=$ $5.1 \cdot 10^{-6} \cdot \mathrm{K}^{-1}$ for the WC phase and $13 \cdot 10^{-6} \cdot \mathrm{K}^{-1}$ for the cobalt phase, and cooling the sample from $750^{\circ} \mathrm{C}$ to room temperature. The temperature of $750^{\circ} \mathrm{C}$ was taken as the approximate temperature were residual stresses may arise. Linear elastic behaviour was assumed for all the temperature range.

\section{$\underline{\text { Results and discussion }}$}

Figure 2 presents the reconstruction in three dimensions of the microstructure of all three materials, showing the WC and metallic phases in darker and lighter colour respectively. As expected, grain size is larger in the Medium grade, with a larger amount of cobalt, and the apparent grain size in the Nanometric grade is much smaller with a relative low 
amount of cobalt. In all grades, cobalt and WC phases are evenly distributed and the microstructure is isotropic in all directions. In the supplementary material, a video presenting the reconstruction is provided.

Microstructure of WC-Co is often described in the literature by four interdependent parameters: Phase volume ( $V_{W C}$ and $\left.V_{C o}\right)$, WC grain size $\left(d_{W C}\right)$, Contiguity $(C)$ and cobalt mean free path $(\lambda)$.

\section{a) Relative volume of each phase}

The relative volume of each phase is one of the most used parameters to define a WC-Co microstructure. Although using weight percent (\%wt) is also common practice, especially in a context of material processing, volume percentage is more informative from the microstructural viewpoint, since $\mathrm{W}$ and $\mathrm{C}$ atoms are dissolved as solid solution within the binder [¡Error! Marcador no definido.].

Once the microstructure has been reconstructed, the relative volumes of each phase (as obtained in the tomographic reconstruction) are compared with the relative volumes estimated from information provided by the manufacturer. These values are presented in table 3. As expected, there is a good agreement with the values supplied by the manufacturer, with the exception of the coarser grade, labelled as Medium. The slight disagreement in values between the 3D reconstructed volume and the provided data can be attributed to the fact that the reconstructed volume is not large enough to provide a homogeneous volume in terms of amount of different phases. In this sense, a simple calculation of the approximate number of WC in each reconstruction can be made. As

indicated in table 2, reconstructed volume of the Medium grade is only $1124 \mu \mathrm{m}^{3}$. Assuming that carbides are spherical with a diameter of $1.64 \mu \mathrm{m}$ (as its size has been measured by linear interception method that assumes this shape), it is obtained that the 
reconstruction volume contains only about 316 carbides, compared to 8,464 for the ultrafine grade and 18,874 for the nanometric scale. The carbide phase is presented in figure 3, where it can be qualitatively appreciated that the number of WC grains is much lower in the Medium grade than in the other two grades.

In figure 4 the carbide phase percentage is presented as a function of the $z$ direction in order to see the scattering of the calculated values. In each point the relative surface of each phase is calculated. As it can be seen, the values calculated for the Ultrafine and Nanometric grains remain stable, with a low scattering, and close to the values estimated from information supplied by the manufacturer. However, the medium grade presents a large scattering depending on the slice number.

All these results show that the volumetric fraction of each phase, as calculated after the 3D reconstruction, yields reliable values as compared to the experimental values, taking into account the scattering on the calculated values for the coarser grade. In this sense, it is worth mentioning that normally the relative volume of both phases is measured by optical measurements of a metallographic surface, yielding the same precision for equivalent areas. Within this context, typical 2D observations generally include around 200 grains [35]; hence, tomography yields at least comparable scattering as the 2D images due to the high density of data acquired in a small volume.

\section{b) Carbide Size}

Carbide size measurements are commonly done by the linear intercept method [17, 18, 24-26]. This methodology may be subjected to some uncertainties due to the irregularity in the shape of the carbides grains, as the correct geometry of each grain cannot be inferred by a single cross section. In this sense, a tomographic reconstruction captures the real geometry of the grains and should provide a real grain size and geometry. In this 
sense, Borgh et al [34] have shown FIB tomographic reconstructions coupled with EBSD which provide reliable data in terms of grain size, shape and orientation. However, this method is time-consuming. Secondary electron images used in this study for 3D reconstruction do not provide orientation contrast; hence, individual grain segmentation through automatic algorithms becomes troublesome. Because of the difficulty of implementing computer-based segmentation on the whole volume, three individual grains of the Medium grade have been manually segmented in order to explore the geometry and size of the grains, as presented in figure 5 .

As it can be seen in this figure, all three of them have a well-faceted triangular prism shape with a truncation on vertices, as described in literature [iError! Marcador no definido.]. WC grains are hexagonal (with two basal (0001) and three prismatic $\{10 \overline{1} 0\}$ planes) and they form well-faceted triangular prism shape with cobalt binder [36, 37]. Since there are two sets of prismatic planes $((10 \overline{1} 0)$ and $(01 \overline{1} 0))$, they tend to form a truncated triangular prism shape, which corresponds to a ditrigonal-bipyramidal class crystal system. Once the carbides have been segmented, the main geometrical parameters are presented in table 4 . The calculated grain size shape corresponds well to the values measured by $2 \mathrm{D}$, with the advantage that the complete geometry of the grain is measured, not just the average grain size.

\section{c) Contiguity}

Contiguity, $C$, is described as the fraction of the interface area of a phase shared by particles of the same phase [2], namely WC, and can be measured as:

$$
1-C=\frac{N_{W C / C o}}{2 \cdot N_{W C / W C}+N_{W C / C o}}
$$


where $N_{W C / W C}$ and $N_{W C / C o}$ are the number of intercepts per unit length of carbide-carbide and carbide-binder boundary respectively. The relation of this parameter with physical properties has been intensively studied [14,16,17]. Experimental values of contiguity show a very wide dispersion $[13,24]$ which can be explained by the influence of carbide size distribution and, in general, decreases with increasing binder content or carbide size. In a plane image, contiguity is measured as a number of intercepts per unit length. For the data obtained by tomography, a similar parameter in three dimensions can be defined that accounts for the relative contact of a carbide grain between binder and neighbouring grains. In this case, instead of the linear carbide-carbide contact, the surface contact should be taken into account. In this sense, we can define the 3D contiguity, $C_{3 D}$, similarly to equation 1 , as:

$$
1-C_{3 D}=\frac{S_{W C / C o}}{2 S_{W C / W C}+S_{W C / C o}}
$$

Where $S_{W C / C o}$ is the relative contact surface between carbide and cobalt, and $S_{W C / W C}$ is the relative contact surface between grains. Whereas $S_{W C / C o}$ can be directly measured form the tomography results, $S_{W C / W C}$ cannot be directly measured if individual carbides are not segmented and individually identified. In our case, segmentation of individual grains could not be done due to the same contrast for each carbide grain orientation, as discussed before, and other techniques, such as EBSD should be used [iError! Marcador no definido.].

Nevertheless, $C_{3 D}$ can be roughly estimated by calculating the average surface of the tungsten carbide grains $\left(S_{T}\right)$, and taking into account that the total surface will be the combination of the surface in contact with the cobalt and with neighbouring grains, as: 


$$
S_{T}=S_{W C / W C}+S_{W C / C o}
$$

The total surface of the grains can be calculated from the measured volume of carbide phase and the estimation of the grain size. From this, the number of carbides per unit volume can be calculated, and knowing the sphericity of the carbides, the total surface of the carbide grains can be estimated.

In table 5 values used for estimating $C_{3 D}$, both the ones measured directly from the tomographic reconstructions as well as the estimated from geometrical considerations, are given. Table 5 also includes the estimated value of $C_{3 D}$ and its comparison to the value measured in two dimensions.

It can be seen that the estimation of $C_{3 D}$ presents higher values than two-dimensional contiguity; however, both values are close, especially for the fine and nanometric grades. Again, the Medium grain is more prone to uncertainty in values due to the fact that the microstructure was quite large for the analysed volume.

It has to be taken into account that surface analysis measures contiguity by linear intercept methods, whereas this method measures the total carbide-carbide contact surface, which is expected to be greater.

In addition, the calculated values of $C_{3 D}$ are an estimation based on assuming single size carbides with a simplified geometry. A more realistic value of $C_{3 D}$ could be measured if segmentation of individual grains can be done by other techniques.

\section{d) Mean free path}


The average separation between carbides is size of binder phase is known as mean free path $(\lambda)$. This parameter can be measured as the apparent grain size of the binder by the Heyn's method as described before.

Because these four parameters are mutually dependant, the relationship between the mean free path and the rest of the abovementioned parameters can be described as: $\lambda=\frac{V_{C o}}{V_{W C}} d_{W C} \frac{1}{1-C}$

Mean free path is defined as the mean length of cobalt between carbides and it is, therefore, a parameter purely based on a surface image, and consequently is a pure 2D parameter.

An equivalent measurement of mean free path in three dimensions should account for the average separation between carbide grains in the metal phase. It has to be recalled that cobalt grains are much larger than the mean free path [37], usually with a size of a few tenths of microns, and therefore the cobalt mean free path is not a parameter of the cobalt phase but of the average separation between carbides.

In order to understand the mean free path in three dimensions, a skeletonization filter to the cobalt phase has been applied, being then able to simplify the shape while maintaining the geometrical and topological properties of the original shape.

Skeletonization algorithms are normally used for pore analysis of rocks, bone or foams [38-42]. Here, skeletonization was applied using a thinning technique, which uses an erosion process that iteratively deletes points from the shape boundary until only onepoint thick curve remains. 
Figure 6 presents the skeleton of the Medium grade together with a fraction of the carbides, showing that indeed the cobalt skeleton remains between the carbide grains. Figure 7 presents the skeletons of the cobalt phase obtained for the three grades studied. Medium grade presents, as expected, a simpler skeleton as compared to the ultrafine and nanometric grades due to the larger microstructure. It is also noteworthy to see that in all three grades the cobalt phase is continuous, that is, that there's a complete percolation of the binder phase, as expected.

Following the above ideas, the skeleton length $\left(\lambda^{3 D}{ }_{\max }\right)$ and the skeleton thickness $\left(\lambda^{3 D} \min \right)$ have been measured. The distance $\lambda^{3 D}{ }_{\max }$ is the maximum straight line of the skeleton. The distance $\lambda^{3 D}$ min is the skeleton thickness, defined as the minimum diameter between the network carbide phases. These two quantities are a measurement of the grain separation, and consequently we define a three-dimensional mean free path $\lambda^{3 D}$ as an average between these two values.

In table 6 the values of these two quantities, together with the average between them $\left(\lambda^{3 D}\right)$ and the mean free path as measured by planar images are presented. In figure 8 the dependence of the dispersion of $\lambda^{3 D}$ max and $\lambda^{3 D}$ on microstructure (binder mean free path) are presented. Values of $\lambda^{3 D}$ and $\lambda$ are in good agreement, which indicates that the $\lambda^{3 D}$ can be taken as the mean free path in $3 \mathrm{D}$.

It is also interesting to note that, not only the mean value is of interest, but also the distribution of maximum (or minimum) free path values for the three hardmetal grades, as seen in figure 8. In this regard, it should be highlighted that relative dispersion of binder mean free path decreases as the microstructure gets coarser (and binder content rises). Assessment of the effective influence of this trend in terms of strength and reliability is not simple, as these mechanical parameters are dependent not only on toughness (intrinsic 
property) but also on nature and size of critical flaws. However, from the viewpoint of toughness, and particularly regarding the corresponding reinforcing mechanism - i.e. toughening at the crack wake due to stretching of ductile ligaments whose thickness is about the binder mean free path - it may be speculated that a narrower dispersion of this two-phase parameter will imply a more homogeneously distributed crack growth resistance (R-curve behaviour [27,43]. Hence, under the simple assumption that critical flaws would be directly related to intrinsic microstructural heterogeneities within the hardmetal grade under consideration, a lower relative deviation of binder mean free path should yield a more effective R-curve behaviour; and thus, an increase in reliability regarding mechanical integrity.

\section{e) Residual stress}

Finally, a simulation of the residual stresses arised during sintering due to thermal expansion mismatch is performed in the Medium grade by finite element modelling. This grade is chosen because being the coarser one, required less elements and, consequenty, less computation time. Nevertheless, the results can be extrapolated to the other grades.

Figure 9 presents the maxium principal stress of the Medium grade, where it can be seen how the carbides are on average in compression and the cobalt in tension. Figure 10 presents a cross section of the middle of the 3D simulation, together with the quantitative tensions across a line containing both carbides and cobalt phase. It is imporant to note that figure 10 is not a 2D simulation, but a cross-section of the 3D simulation, and hence reflects the $3 \mathrm{D}$ results.

The average residual stress across the volume is around -475MPa for the carbide particles and around $1200 \mathrm{MPa}$ for the cobalt phase. This is in reasonable agreement with the experimental values reported by Krawitz and Drake [44], with values of aprox 800MPa 
for the cobalt phase and -600MPa for the WC phase, considering a medium grade of $47 \%$ Volume Co, relatively similar to the Medium grade presented here.

It is interesting to highlight, not only the good agreement in the average values of residual stress, but also how these residual stresses are distributed inside the different particles. As it can be seen in figure 10, the residual stress is maximum in the center of the WC particles (up to $-600 \mathrm{MPa}$ ) and decreases towards the boundary. In some locations of the WC particles, the residual stress even becomes tensile (see arrows in figure 10), indicating that some regions of the WC particles are in tension although the mean values of the residual stress is compressive. This is in complete agreement with the local stress state found at some WC angularities by Livescu et al.[45]. Due to the brittle-like nature of the WC particles, these regions subjected to tensile stresses are suceptible to generating cracks under loads lower than expected, affecting thus to the reliability of the material.

\section{Conclusions}

Focused Ion Beam tomography on three different WC-Co grades has been successfully performed. The 3D reconstructions provide a more clarifying view of the microstructure of the material as well as to serve as inputs for modelling, proving that FIB tomography is a powerful technique to gain insight into geometrically complex materials as cemented carbides. From the obtained results, the following conclusions can be made:

1) Percentages of phases can reliably be extracted from tomography, provided that the volume reconstructed is representative enough of the material. In this sense, the Medium grade had a larger scatter in the results because of the relatively small amount of material sampled.

2) Estimation of grain size and contiguity has also been satisfactorily assessed. However, further improvement requires individual segmentation of grains, 
depending on their crystallographic orientation. This was not possible to accomplish with FIB/SEM tomography.

3) Mean free path in three dimensions yields values close to the $2 \mathrm{D}$ values. and it provides a better look on how the cobalt is distributed as a 3D network. In this sense, we were able to visualize the percolation of the binder phase as to see the statistical variability of the separation between carbides.

4) Residual stresses were modelled using a simple elastic model, yielding average results comparable to the ones reported experimentally. The use of a real microstructure mesh, resulting as direct outcome of 3D image reconstruction of FIB tomography, allowed to discern details on the residual stress distribution. In particular, it was found that some areas of the WC can be in tension, despite that the average values in this phase are in compression. This level of detail is seldom achivable with a experimental technique. The same microstructures obtained here can serve as imputs for more complex models, using real geometries of microstructures and under different loading conditions.

\section{Acknowledgments}

This work was financially supported by the Spanish Ministerio de Economía y Competitividad (Grant MAT2015-70780-C4-3-P). Additionally, J.M. Tarragó acknowledges the Ph.D. scholarship received from the collaborative Industry-University program between Sandvik Hyperion and Universitat Politècnica de Catalunya.

\section{Biblography}

1. H.E. Exner, Physical and chemical nature of cemented carbides, Int. Met. Rev. 4 (1979) 149-173. 
2. J. Gurland, New scientific approached to development of tool materials, Int. Mater. Rev. 33 (1988) 151-166

3. L. Prakash, Fundamentals and general applications of hardmetals, in Comprehensive Hard Materials, Eds. V.K. Sarin, D. Mari and L. Llanes, Elsevier, UK (2014) pp. 29-90

4. H.F. Fischmeister, Development and present status of the science and technology of hard materials, in Science of Hard Materials, Eds. R.K. Viswanatham, D.J. Rowcliffe and J. Gurland, Plenum Press, New York, USA (1981) pp. 1-45.

5. R.K. Viswanadham, T.S. Sun, E.F. Drake and J.A. Peck, Quantitative fractography of WC-Co cermets by Auger spectroscopy, J. Mater. Sci. 16 (1981) 1029-1038.

6. L.S. Sigl and H.E. Exner, Experimental study of the mechanics of fracture in WCCo alloys, Metall. Trans. 18A (1987) 1299-1308

7. L.S. Sigl and H.F. Fischmeister, On the fracture toughness of cemented carbides, Acta Metall. 36 (1988) 887-897

8. B. Roebuck and E.A. Almond, Deformation and fracture processes and the physical metallurgy of WC-Co hardmetals, Int. Mater. Rev. 33 (1988) 90-112

9. D.G.F. O’Quigley, S. Luyckx and M.N. James, An empirical ranking of a wide range of WC-Co grades in terms of their abrasion resistance measured by the ASTM standard B 611-85 test, Int. J. Refract. Met. Hard Mater. 15 (1997) 73-79

10. L. Makhele-Lekala, S. Luyckx, and F.R.N. Nabarro, Semi-empirical relationship between the hardness, grain size and mean free path of WC-Co, Int. J. Refract. Met. Hard Mater. 19 (2001) 245-249

11. L. Llanes, Y. Torres and M. Anglada, On the fatigue crack growth behavior of WC- Co cemented carbides: kinetics description, microstructural effects and fatigue sensitivity, Acta Mater. 50 (2002) 2381-2393

12. L.J. Prakash, Development in tungsten carbide-cobalt cemented carbides, Int. Powder Met. (2009) 131-144.

13. J.M. Tarragó, D. Coureaux, Y. Torres, D. Casellas, I. Al-Dawery, L. Schneider and L. Llanes, Microstructural effects on the R-curve behavior of WC-Co cemented carbides, Mater. Design 97 (2016) 492-501

14. J. Gurland, The measurement of grain contiguity in two-phase alloys, Trans. Metal. Soc. AIME 212 (1958) 452-455

15. B. Roebuck and G. Bennett, Phase size distribution in WC/Co hardmetal, Metallography 19 (1986) 27-47

16. A. V. Shatov, S. A. Firstov, and I. V. Shatova, The shape of WC crystals in cemented carbides, Mat. Sci. Eng. A 242 (1998) 7-14

17. S.Y. Kang, J.G. Roemer and D. Ghosh, Microstructural characterization of cemented carbide samples by image analysis techniques, Powder Technol. 108 (2000) 130-136.

18. H. Engqvist and B. Uhrenius, Determination of the average grain size of cemented carbides, Int. J. Refract. Met. Hard Mater. 21, (2003) 31-35 
19. S. Luyckx and A.D. Love, Empirical quantitative relationships among grain size, mean free path, contiguity and cobalt content in WC-Co hardmetal, Trans. Royal Soc. South Africa 58 (2003) 145-148.

20. V.T. Golovchan and N.V. Litoshenko, On the contiguity of carbide phase in WCCo hardmetals, Int. J. Refract. Met. Hard Mater. 21 (2003) 241-244.

21. C.-S. Kim and G.S. Rohrer, Geometric and crystallographic characterization of WC surfaces and grain boundaries in WC-Co composites, Interface Sci. 12 (2004) 19-27.

22. A.V. Shatov, S.S. Ponomarev, S.A. Firstov and R. Warren, The contiguity of carbide crystals of different shapes in cemented carbides, Int. J. Refract. Met. Hard Mater. 24 (2006) 61-74

23. S. Luyckx and A.D. Love, The dependence of the contiguity of WC on Co content and its independence from WC grain size in WC-Co alloys, Int. J. Refract. Met. Hard Mater. 24 (2006) 75-79

24. J.M. Tarragó, D. Coureaux, Y. Torres, F. Wu, I. Al-Dawery and L. Llanes, Implementation of an effective time-saving two-stage methodology for microstructural characterization of cemented carbides, Int. J. Refract. Met. Hard Mater. 55 (2016) 80-86

25. M. Brieseck, W. Languer, B. Geneib, K. Wagner and S. Wagner, A straightforward method for analysing the grain-size distribution in tungsten carbide - cobalt hardmetals, Microchim. Acta 168 (2010) 309-316

26. B. Roebuck, K.P. Mingard, H. Jones and E.G. Bennett, Aspects of the metrology of contiguity measurements in WC based hard materials, Int. J. Refract. Met. Hard Mater. 62 (2017) 161-169

27. J.M. Tarragó, E. Jiménez-Piqué, L. Schneider, D. Casellas, Y. Torres, L. Llanes, FIB/FESEM experimental and analytical assessment of R-curve behavior of WCCo cemented carbides, Mat. Sci. Eng. A 645 (2015) 142-149

28. J.M. Tarragó, E. Jimenez-Pique, M. Turon-Vinas, L. Rivero, I. Al-Dawery, L. Schneider, and L. Llanes, Fracture and fatigue behavior of cement carbides: 3D focused ion beam tomography of crack-microstructure interactions, Int. J. Powder Metall. 50 (2014) 33-42

29. J.M. Tarragó, G. Fargas, E. Jiménez-Piqué, A. Felip, L. Isern, D. Coureaux, J.J. Roa, I. Al-Dawery, J. Fair and L. Llanes, Corrosion damage in WC-Co cemented carbides: Residual strength assessment and 3D FIB-FESEM tomography characterization, Powder Metall. 57 (2014) 324-330

30. J.M. Tarragó, G. Fargas, L. Isern, S. Dorvlo, E. Tarres, C.M. Müller, E. JiménezPiqué and L. Llanes, Microstructural influence on tolerance to corrosion-induced damage in hardmetals, Mater. Design 111 (2016) 36-43

31. A. Velichko, C. Holzapfel, A. Siefers, K. Schladitz, F. Mücklich, Unambiguous classification of complex microstructures by their three-dimensional parameters applied to graphite in cast iron, Acta Mater. 56 (2008) 1981-1990.

32. E. Jiménez-Piqué, A. Ramos, J.A. Muñoz-Tabares, A. Hatton, F. Soldera, F. Mücklich and M. Anglada, Focused ion beam tomography of zirconia degraded under hydrothermal conditions, J. Eur. Ceram. Soc. 32 (2012) 2129-2136. 
33. N. Cuadrado, J. Seuba, D. Casellas, M. Anglada and E. Jiménez-Piqué, Geometry of nanoindentation cube-corner cracks observed by FIB tomography: Implication for fracture resistance estimation, J. Eur. Ceram. Soc. 35 (2015) 2949-2955

34. I. Borgh, P. Hedström, J. Odqvist, A. Borgenstam, J. Agren, A. Gholinia, B. Winiarski, P.J. Withers, , G.E. Thompson, K. Mingard, M.G. Gee, On the threedimensional structure of WC grains in cemented carbides, Acta Mater. 61 (2013) 4726-4733

35. E. G. Bennet and B. Roebuck "The metallographic Measurement of Hardmetal Grain Size” NPL Measurement Good Practice., 22 (2000). Edited by National Physical Laboratory, Teddington, UK.

36. A. V. Shatov, S. A. Firstov, and I. V. Shatova, "The shape of WC crystals in cemented carbides,” Materials Science and Engineering: A, vol. 242, no. 1-2, pp. 7-14, 1998.

37. K.P. Mingard, B. Roebuck, J. Marshall and G. Sweetman, Some aspects of the structure of cobalt and nickel binder phases in hardmetals, Acta Mater. 59 (2011) 2277-2290

38. L. Holzer, F. Indutnyi, P. H. Gasser, B. Munch, and M. Wegmann, Threedimensional analysis of porous BaTiO3 ceramics using FIB nanotomography., J. Microscopy 216 (2004) 84-95.

39. L. Pothuaud, P. Porion, E. Lespessailles, C. L. Benhamou, and P. Levitz, “A new method for threedimensional skeleton graph analysis of porous media: Application to trabecular bone microarchitecture," Journal of Microscopy, vol. 199, no. Pt 2, pp. 149-61, 2000

40. G. Aufort, R. Jennane, R. Harba, and C. L. Benhamou, "A New 3D ShapeDependent Skeletonization Method. Application to Porous Media," in 14th European Signal Processing Conference (EUSIPCO), 2006.

41. W. B. Lindquist and A. Venkatarangan, "Investigating 3D Geometry of Porous Media from High Resolution Images,” Physics and Chemistry of Earth, Part A, vol. 25, no. 7, pp. 593-599, 1999.

42. R. I. Al-Raoush and C. S. Willson, "Extraction of physically realistic pore network properties from three-dimensional synchrotron X-ray microtomography images of unconsolidated porous media systems,” Journal of Hydrology, vol. 300, no. 1-4, pp. 44-64, 2005.

43. J.M. Tarragó, D. Coureaux, Y. Torres, D. Casellas, I. Al-Dawery, L. Schneider and L. Llanes, Microstructural effects on the R-curve behavior of WC-Co cemented carbides Mater. Design 97 (2016) 492-501

44. A. Krawitz and E. Drake "Residual stresses in cemented carbides- An overview" nt. Journal of Refractory Metals and Hard Materials 49 (2015) 27-35

45. V. Livescu, B. Clausen, J.W. Paggett, A.D. Krawitz, E.F. Drake, and M.A.M. Bourke, Measurement and modeling of room temperature Co-deformation in WC-10 wt.\% Co Mater. Sci. Eng. A 399 (2005) 134-140 
